\title{
CARACTERIZACIÓN DEL REGISTRO CERÁMICO DEL SITIO ARQUEOLÓGICO FAMILIA PRIMÓN (CORONDA, SANTA FE, ARGENTINA): DIVERSIDAD DE LÍNEAS DE ABORDAJE
}

\author{
Characterization of pottery record from Familia Primón archaeological site \\ (Santa Fe, Argentina): diversity of approach lines
}

\author{
Fernando Balducci* \\ https://orcid.org/0000-0001-9890-059X \\ Paula Galligani** \\ https://orcid.org/0000-0002-3949-5333 \\ Julieta Sartori*** \\ https://orcid.org/0000-0001-6684-5039
}

\section{Resumen}

En este trabajo se presentan los resultados del análisis del material cerámico recuperado en dos sectores del sitio arqueológico Familia Primón (Coronda, Santa Fe). Se identificaron una serie de variables tafonómicas con el objetivo de evaluar los procesos postdepositacionales que pudieron haber afectado la preservación de los conjuntos y, posteriormente, se relevaron diferentes atributos tecnomorfológicos y decorativos. Las características observadas ubican a la alfarería de FP en lo que se denomina contexto Goya-Malabrigo aunque, por otro lado, se identificaron rasgos vinculados a la unidad arqueológica Guaraní, poco frecuente en el área de estudio. Se discuten los resultados en relación con los materiales recuperados en otros sitios del área y se espera que la información obtenida contribuya al conocimiento de la tecnología cerámica de los grupos originarios que habitaron el centro-este de la provincia de Santa Fe durante el período de contacto europeo-indígena.

Abstract

$$
<\text { Alfarería }><\text { Nordeste argentino }><\text { Familia Primón }><\text { Arqueología }>
$$

This paper presents the results of pottery analysis of two areas from the Familia Primón archaeological site (Coronda, Santa Fe). A series of taphonomic attributes were identified in order to evaluate several postdepositional processes which could have affected the preservation of both assemblages. Then different tecnomorphological and decorative attributes were detected. The observed features locate the pottery of FP in what is called a Goya-Malabrigo context, although, on the other hand, characteristics related to the Guaraní archaeological unit were identified, which is rare in the study area. The results are discussed in relation to the material record recovered in other regional archaeological sites; it is expected that the information obtained will contribute to knowledge about ceramic technology of indigenous people of central-eastern Santa Fe province, during the european-indigenous contact period.

$$
<\text { Pottery }><\text { Northeastern Argentina }><\text { Primón Family }><\text { Archaeology }>
$$

Recibido: 01/10/2018//Aceptado: 23/12/2018

* Fundación Arqueológica del Litoral (FUNDARQ). Grupo de Investigaciones Arqueológicas del Nordeste (GIAN). Rosario, (Santa Fe), Argentina, Doctorando en Ciencias Antropológicas en la Universidad Nacional de Córdoba, Argentina, ferbalducci@gmail.com

** Fundación Arqueológica del Litoral (FUNDARQ). Grupo de Investigaciones Arqueológicas del Nordeste (GIAN). Rosario, (Santa Fe), Argentina, Becaria doctoral del Consejo Nacional de Investigaciones Científicas y Técnicas (CONICET), Argentina, paulagalligani@hotmail.com

***Fundación Arqueológica del Litoral (FUNDARQ). Grupo de Investigaciones Arqueológicas del Nordeste (GIAN). Rosario, (Santa Fe), Argentina, Investigadora Asistente del Consejo Nacional de Investigaciones Científicas y Técnicas (CONICET), Argentina, julisartori@gmail.com 
Balducci, Galligani y Sartori. Caracterización del registro cerámico del sitio arqueológico Familia Primón...

\section{Introducción}

Desde sus inicios, la arqueología del Nordeste argentino — particularmente del área vinculada con la cuenca del río Coronda-, se centró en el registro cerámico, en su descripción estilística y morfológica, con el objetivo de establecer similitudes y diferencias que pudieran ser adscriptas a determinados tipos culturales (Serrano, 1923; Iribarne, 1937; Badano, 1940, entre otros). En base a estas descripciones, se elaboraron grandes sistematizaciones que intentaron explicar el desarrollo histórico de ocupación de la región, agrupando determinadas características de la alfarería y, en menor medida, de la tecnología (ósea y lítica) e inhumaciones. Las mismas eran asignadas a una cultura particular, ubicada en un espacio y en un momento determinados (Lafón, 1972; Serrano, 1972; Ceruti, 1986).

Con el cambio de paradigma que se dio en la arqueología regional hacia finales de la década de 1990, los análisis cerámicos de la zona comenzaron a abordar, desde distintos marcos teóricos e interpretativos, diferentes problemáticas vinculadas no sólo a los aspectos decorativos (v.g. Balducci et al., 2017; Di Prado, 2016; Galligani y Balducci, 2014; Ottalagano, 2010, 2015), sino también cuestiones relacionadas con la tecnomorfología de los contenedores (v.g. Feuillet Terzaghi et al., 2009; Letieri, 1999; Ottalagano, 2016; Píccoli y Barboza, 2013; Sartori et al., 2013) o los procesos postdepositacionales que influyen en su preservación (v.g. Bonomo et al., 2017; Cocco, 1996; Escudero, 1996; Galligani et al., 2018). De este modo, la alfarería comenzó a ser integrada a preguntas de investigación más amplias y los análisis se complementaron con otros estudios, para dar respuesta a interrogantes acerca del modo de vida y las prácticas de subsistencia de las poblaciones que habitaron la región en el pasado.

Sin embargo, si bien la zona del río Coronda cuenta con trabajos sistemáticos desde hace una década (Balducci, 2014; Feuillet Terzaghi, 2009; Sartori, 2008, 2013), los estudios específicos sobre alfarería fueron locales (i.e. a nivel de sitio arqueológico) y sólo algunos intentaron realizar comparaciones inter-sitio (Galligani et al. 2018; Sartori et al. 2013) aunque no desde una perspectiva regional. En este sentido, el proyecto "Análisis arqueológico integral de la tecnología cerámica de cuencas fluviales del centroeste santafesino (ríos Coronda y Salado del norte)", llevado adelante por uno de los autores de este trabajo (FB), considera que las elecciones humanas sobre la tecnología cerámica están mediadas por distintas variables (v.g. ambiental, social, simbólico) y que, en consecuencia, existen diferencias en las características de la alfarería que estarían vinculadas con las diversas estrategias de subsistencia y "modos de hacer" de los grupos humanos que habitaron en el pasado (v.g. Rice 1987; Lemonnier 1992).

Este trabajo, que se enmarca dentro de dicho proyecto, tiene como objetivo ampliar el conocimiento sobre la alfarería de los grupos originarios que habitaron el centro-este de la provincia de Santa Fe durante el período de contacto europeo-indígena. Para contribuir a tal fin, se considera que es necesario profundizar en distintos aspectos del registro recuperado en los sitios arqueológicos del área (v.g. tecnomorfología, procesos postdepositacionales) para luego integrar los resultados a nivel regional (Galligani et al., 2018; Sartori et al., 2013). En esta instancia, se presenta una primera 
caracterización de los conjuntos cerámicos recuperados en el sitio arqueológico Familia Primón, ubicado sobre la margen derecha del río Coronda, específicamente en los sectores FP2 y FP3, excavados en los años 2013 y 2014, respectivamente. Se analizaron variables tecnomorfológicas y decorativas de los restos cerámicos, luego de evaluar la potencial incidencia de agentes tafonómicos, para poder comparar ambos conjuntos sobre una base más sólida. Los resultados obtenidos se discuten en el marco de las investigaciones que se vienen llevando a cabo en otros sitios de la zona y en relación con la alfarería hallada en la región del Nordeste argentino.

\section{El sitio arqueológico Familia Primón}

El sitio arqueológico Familia Primón (FP) se encuentra en el centro-este de la provincia de Santa Fe (Argentina), zona de transición biogeográfica entre las ecorregiones del Espinal y del Delta e Islas del Paraná (sensu Burkart et al., 1999), que presenta rasgos de los bosques fluviales del río Paraná y de aquellos que corresponden a la llanura chaco-pampeana. El sitio se ubica en la zona sur de la ciudad de Coronda, en un área elevada con respecto a la planicie de inundación de la margen derecha del río homónimo (Figura 1).

Figura 1. Ubicación de la localidad arqueológica Familia Primón (Coronda, Santa Fe).
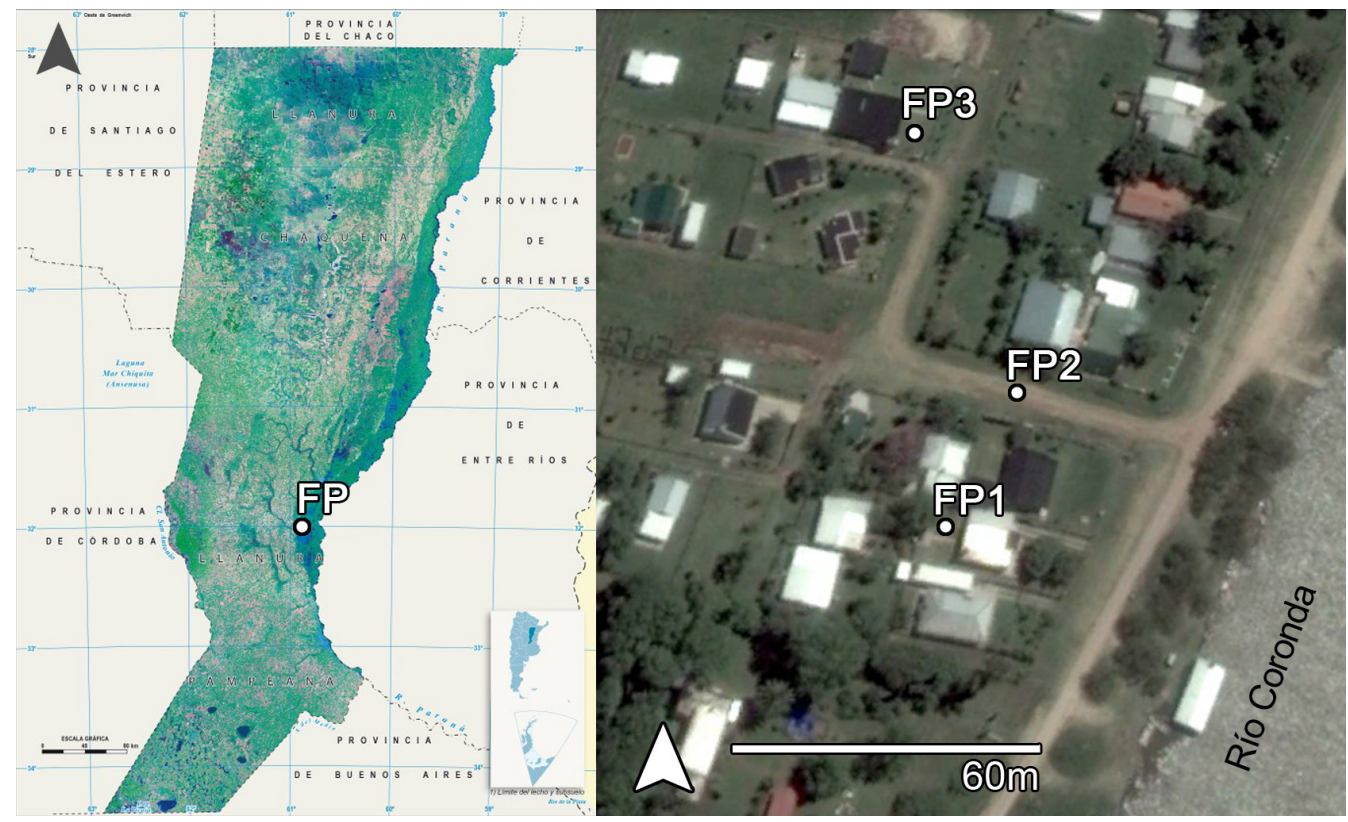

Familia Primón se compone de tres sectores, excavados en diferentes etapas, que reflejan distintas áreas de actividad, cuyos fechados radiocarbónicos indican un único momento de ocupación (ver infra). El área de entierros (de aquí en adelante sector FP1) fue localizada durante la remodelación del patio de una vivienda particular en 
Balducci, Galligani y Sartori. Caracterización del registro cerámico del sitio arqueológico Familia Primón...

el año 2004 (Figura 1). Se excavaron ocho cuadrículas de 2x2 m cada una, cubriendo una superficie de $32 \mathrm{~m}^{2}$, hallándose entierros primarios y secundarios y, además, una significativa cantidad de fragmentos cerámicos, restos faunísticos y escasos desechos de material lítico (Feuillet Terzaghi, 2009).

En diciembre de 2013, tras la apertura de una calle a 30 metros de FP1, quedaron expuestos gran cantidad de materiales arqueológicos. En consecuencia, se realizaron recolecciones superficiales sistemáticas en un área de $6 \mathrm{~m}^{2} \mathrm{y}$, posteriormente, se trazaron 2 cuadrículas de $2 \times 2 \mathrm{~m}$ y $30 \mathrm{~cm}$ de profundidad. A este sector se lo denominó FP2 (Figura 1), recuperándose gran cantidad de tiestos cerámicos, elementos líticos y restos óseos faunísticos (Balducci, 2014; Galligani y Balducci, 2014).

A comienzos de 2014 se llevó a cabo una excavación sistemática en un predio privado, donde luego se construyó una vivienda, a $60 \mathrm{~m}$ de FP2. Se trazaron tres cuadrículas de $2 \times 1 \mathrm{~m}$, las cuales fueron excavadas por niveles artificiales de $5 \mathrm{~cm}$ y cubrieron un total de $5.50 \mathrm{~m}^{2}$ aproximadamente, alcanzando profundidades de $80 \mathrm{~cm}$ en promedio. Asimismo, se plantearon 4 pozos de sondeo de 1x $1 \mathrm{~m}$, en los cuales se recuperaron escasos materiales arqueológicos. En general, en este sector se hallaron gran cantidad de fragmentos cerámicos y restos óseos faunísticos, además de escasos artefactos líticos y macrorrestos vegetales (Balducci, 2014; Galligani y Balducci, 2014; Sartori et al., 2018). Este sector se denominó FP3 (Figura 1) y es el que exhibe menor perturbación antrópica actual, si se lo compara con los otros dos sectores, ya que el registro presenta una mayor integridad, lográndose recuperar escamas de pescado y restos de moluscos que son de alta fragilidad (Sartori, 2013). Por otra parte, la gran cantidad de tiestos cerámicos pertenecientes a una misma cuadrícula que pudieron ser remontados, refleja una baja dinámica interna del depósito.

El sitio cuenta con dos dataciones radiocarbónicas: una de $370 \pm 30$ años AP (UGAMS 02471) — realizada sobre un diente humano extraído de un individuo ubicado a $70 \mathrm{~cm}$ de profundidad en FP1 (Sartori, 2008) - y otra de 470 \pm 50 años AP (LP-3037) - efectuado sobre una muestra de sedimentos tomada de FP3, entre 27,5 y $44,5 \mathrm{~cm}$ de profundidad, estrato donde se halló la mayor cantidad de materiales arqueológicos(Balducci, 2014). El cálculo de la media ponderada (Ward y Wilson, 1978) dio como resultado que las edades radiocarbónicas disponibles son estadísticamente indistinguibles (g.l. $=1 ; \mathrm{T}=2,94 ; \mathrm{x}^{2}=3,84 ; \mathrm{p}>0,05$ ), arrojando un valor ponderado y una desviación estándar de 396 \pm 26 años radiocarbónicos AP y el rango de edades calibradas, con una probabilidad del 95\%, es de 1456-1626 años cal AD $^{1}$ (Balducci, 2014). En síntesis, el marco cronológico del sitio lo sitúa en momentos de contacto europeo-indígena, lo que lo constituye en uno de los escasos registros para este período en la zona.

\section{Materiales y Métodos}

Para este trabajo se seleccionaron los materiales cerámicos recuperados en los sectores FP2 y FP3, excluyéndose aquellos fragmentos menores a $2 \mathrm{~cm}$ de longitud, dado

\footnotetext{
${ }^{1}$ La calibración de los fechados se efectuó mediante la curva SHCal-13 (Hogg et al., 2013), utilizando el programa Calib Rev 7.0.2 (Stuiver et al., 2005).
} 
que no permitieron la observación de la mayoría de los atributos a relevar. Los conjuntos analizados se componen de 719 tiestos en FP2 y de 1328 en FP3. Todos los fragmentos de FP3 proceden de estratigrafía, mientras que en FP2, el 28\% $(n=201)$ provienen de superficie. No obstante, al no existir diferencias estadísticamente significativas entre los resultados de los análisis realizados sobre los fragmentos de estratigrafía y de superficie (excepto la variable inclusiones), los tiestos de FP2 fueron tratados en conjunto, sin diferenciarlos de acuerdo con su procedencia.

Dado que, al igual que los demás ítems del registro arqueológico, los fragmentos cerámicos se hallan sujetos a la acción de agentes y procesos postdepositacionales (Tschegg, 2009), en primera instancia se evaluaron una serie de variables vinculadas con la preservación de los mismos, tales como: tipos y grados de abrasión (Schiffer y Skibo, 1989) —entre los cuales se distingue el efecto pedestal (Sanhueza Riquelme, 1998; Skibo, 1987; Skibo y Schiffer, 1987), agrietamiento (Amitrano Bruno, 1984; Schiffer y Skibo, 1989) y delaminación (Schiffer y Skibo, 1989)—. También se relevó el grado de redondeamiento (Sanhueza Riquelme, 1998), presencia/ausencia de residuos orgánicos (Beck et al., 2002; García Rosselló y Calvo Trias, 2006; Hally, 1983; Skibo, 1992), óxido de manganeso - en forma de puntos o manchas de color marrón-negro en la superficie - (Bennàsar Serra, 2010), sales (Fantuzzi, 2010), marcas de raíces - en patrón dendrítico que tiñe y/o corroe la superficie- (Barrientos, 1997; Behrensmeyer, 1978; Gutiérrez, 2004) y roedores - localizadas exclusivamente en los bordes de los tiestos, similar a como suelen presentarse en los restos óseos- (Gutiérrez, 2004). La observación de los diferentes aspectos se realizó macroscópicamente y, en los casos que así lo requirieron, mediante el uso de una lupa binocular Mikoba 745 (50X).

Posteriormente, se asignó cada fragmento a una parte específica del contenedor, de acuerdo a los criterios establecidos por Balfet y colaboradores (1992): cuerpo, borde, base y apéndices. Además, se registraron aspectos que aportan información sobre la atmósfera de cocción de las piezas, su impermeabilidad, maleabilidad, entre otros: inclusiones, fractura y color - tanto en las superficies y en los márgenes (internosexternos) como en el núcleo de cada tiesto- (Banning, 2000; Convención Nacional de Antropología, 1966; Cremonte y Bugliani, 2006-2009; Revised Standard Soil Color Charts, 2001; Rice, 1987; Rye, 1981). Para el caso de las fracturas, dada la cantidad de materiales que componen ambos conjuntos, se decidió tomar una muestra aleatoria, de aproximadamente el 20\% de cada uno de ellos, para su relevamiento, fracturándose 164 tiestos de FP2 y 290 de FP3.

También se relevaron una serie de variables vinculadas con el tratamiento general de las superficies que, en sentido amplio, son relativas a aspectos decorativos y funcionales: baño/engobe, alisado, pulido, pintura, incisión, unguiculado, corrugado y otros tratamientos (v.g. cepillado, roletado) (Convención Nacional de Antropología, 1966; Cremonte y Bugliani, 2006-2009; González y Frère, 2010). Esto se realizó para evaluar la variabilidad que exhibe el conjunto respecto a las diferentes técnicas o estilos relevados para el área según los antecedentes bibliográficos y que, tradicionalmente, han sido asignados a diferentes grupos culturales o "entidades culturales" (sensu Ceruti, 1986, 2003). 
Con el objetivo de comparar la variabilidad entre los conjuntos, se realizaron pruebas estadísticas adaptadas a la evaluación de hipótesis de nulidad $(\alpha=0,01)$. Las diferencias en las proporciones de casos positivos para las distintas variables registradas en cada uno de los conjuntos cerámicos analizados fueron evaluadas, estadísticamente, mediante una prueba de $\chi^{2}(1$ g.l., $\alpha<0,01)$. Los análisis estadísticos se llevaron a cabo mediante el uso del software Infostat (versión 2016).

\section{Resultados}

En relación con las partes de las vasijas, en ambos sectores los fragmentos fueron asignados, en mayor proporción, a la categoría cuerpo ( $\mathrm{FP} 2=83,46 \%, \mathrm{FP} 3=82,32 \%$ ), seguidos por bordes $(\mathrm{FP} 2=14,32 \%, \mathrm{FP} 3=14,83 \%) \mathrm{y}$, con menor frecuencia, bases (FP2= $1,39 \%, \mathrm{FP} 3=2,03 \%$ ) y apéndices $(\mathrm{FP} 2=0,83 \%, \mathrm{FP} 3=0,82 \%)$. Dentro de estos últimos, se identificaron asas $(\mathrm{FP} 2=5, \mathrm{FP} 3=7)$, zoomorfos $(\mathrm{FP} 3=2)$, mamelonares $(\mathrm{FP} 2=1$, $\mathrm{FP} 2=1)$ y recortados $(\mathrm{FP} 3=1)$. Respecto de los apéndices zoomorfos, vale mencionar que se hallan representados un ave (Psittacidae) y un mamífero (Atelidae) (Figura 2). Asimismo, se identificaron escasos restos de alfarería gruesa o campana (sensu Gaspary, 1950) (5 fragmentos en cada sector).

Figura 2. Apéndices zoomorfos recuperados en FP3: A: Psittacidae; B: Atelidae.

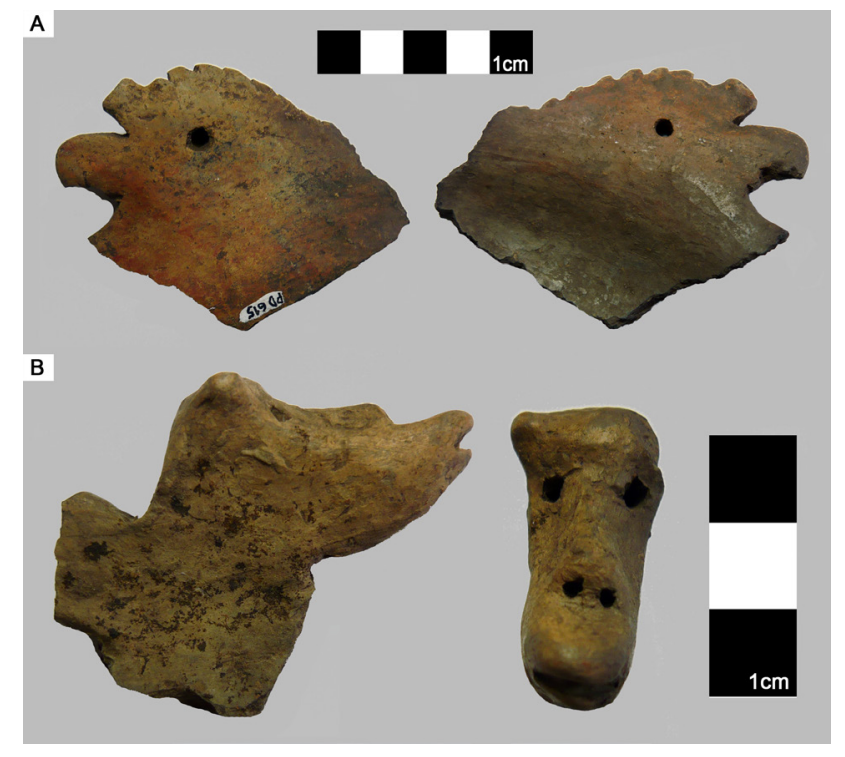

En cuanto a los factores tafonómicos, ambos sectores se vieron afectados por todos los atributos relevados, aunque con algunas diferencias de intensidad. En FP2 se observaron marcas de raíces en un $12,93 \%(\mathrm{n}=93)$, mientras que las trazas generadas por roedores se relevaron sólo en un $0,83 \%(\mathrm{n}=6)$. Acerca de las depositaciones químicas, el manganeso afectó la muestra en un 31,71\% ( $\mathrm{n}=228)$ y el hollín en un 10,29\% ( $\mathrm{n}=74)$. 
Los procesos de abrasión influyeron sobre el conjunto, relevándose efecto pedestal en un $7,79 \%(n=56)$, agrietamiento en un $11,68 \%(n=84)$, delaminación en un $14,19 \%$ $(n=102)$ y redondeamiento en un $11,27 \%(n=81)$. Por su parte, en FP3 se registró la presencia de marcas de raíces en un $8,66 \%(n=115)$ y de roedores en un $2,48 \%(n=33)$, mientras que las depositaciones de manganeso fueron observadas en un $24,4 \%(n=324)$ y las de hollín en un 10,54\% $(\mathrm{n}=140)$. Éstas últimas se relevaron en la cara externa de los tiestos, al igual que en FP2, aspecto que podría estar vinculado con la cocción de los alimentos. En relación a los procesos abrasivos, se relevó efecto pedestal en un $7,45 \%(n=9)$, agrietamiento en un 5,05\% $(n=67)$ y delaminación y redondeamiento en un $11,37 \%(\mathrm{n}=151)$ y en un $11,45 \%(\mathrm{n}=152)$, en cada caso (Tabla 1$)$.

Tabla 1. Frecuencia de las distintas variables tafonómicas para FP2 y FP3. En rojo las variables que presentan diferencias significativas entre los conjuntos.

\begin{tabular}{lccccc}
\hline \multirow{2}{*}{ Variables tafonómicas } & \multicolumn{2}{c}{$\mathrm{FP} 2(\mathrm{~N}=719)$} & $\mathrm{FP} 3(\mathrm{~N}=1328)$ & \multirow{2}{*}{$\mathrm{X}^{2}$} \\
\cline { 2 - 5 } & $\mathrm{n}$ & $\%$ & $\mathrm{n}$ & $\%$ & \\
\hline Roedores & 6 & 0,83 & 33 & 2,48 & $\mathrm{p}=0,0091$ \\
\hline Raíces & 93 & 12,93 & 115 & 8,66 & $\mathrm{p}=0,0022$ \\
\hline Ó. de Manganeso & 228 & 31,71 & 324 & 24,4 & $\mathrm{p}<0,0004$ \\
\hline Hollín & 74 & 10,29 & 140 & 10,54 & $\mathrm{p}=0,8599$ \\
\hline Pedestal & 56 & 7,79 & 99 & 7,53 & $\mathrm{p}=0,7852$ \\
\hline Agrietamiento & 84 & 11,68 & 67 & 5,12 & $\mathrm{p}<0,0001$ \\
\hline Delaminación & 102 & 14,19 & 151 & 11,45 & $\mathrm{p}<0,0646$ \\
\hline Redondeamiento & 81 & 11,27 & 152 & 11,52 & $\mathrm{p}=0,9025$ \\
\hline
\end{tabular}

Los resultados de las variables vinculadas con los tratamientos superficiales se presentan en forma sintética en la Tabla 2. En FP3 se observan mayores porcentajes de baño y pulido que en FP2, mientras que el alisado en este último es levemente superior. Por otra parte, son similares las frecuencias de corrugado y de unguiculado en ambos conjuntos (Figuras 3c y 3e). Respecto de la decoración incisa, se registró en un 5,42\% $(n=39)$ en FP2 y en un 3,92\%, $(n=52)$ en FP3, estando mayormente representada en el labio del borde en ambos casos ( $n=27$ en FP2 y $n=43$ en FP3). En lo que refiere al tipo de incisión, en los bordes prevalece el inciso de línea ( $n=33$ en FP3 y n=21 en FP2), seguido inciso de punto ( $\mathrm{n}=10$ en FP3 y $n=6$ en FP2), mientras que en los cuerpos, predomina el surco rítmico ( $n=10$ en FP2 y $n=5$ en FP3), seguido de línea ( $n=5$ en FP2 y $n=4$ en FP3) y de punto ( $n=2$ en FP2). En relación a la presencia de pintura, si bien fue relevada en ambos sectores, representa el doble en FP3 $(n=209)$ que en FP2 $(n=56)$. En ambos conjuntos es mayoritario el color rojo, comúnmente en la parte interna de los fragmentos, ya sea como bandas paralelas al borde o con motivos geométricos; con algunas excepciones ( $n=8$ en FP3 y $n=3$ en FP2) que exhiben pintura blanca de fondo con líneas delgadas rojas (Figura 3a). Por último, dentro de la categoría "otras", se registraron variables como cepillado y roletado, aunque en bajos porcentajes (Figura $3 \mathrm{~b}$ y 3d) (Tabla 2). 


\section{ARTÍCULOS}

Balducci, Galligani y Sartori. Caracterización del registro cerámico del sitio arqueológico Familia Primón...

Figura 3. Cerámica con tratamiento superficial recuperada en la localidad arqueológica FP: a) pintura roja sobre blanca; b) roletado; c) corrugado; d) cepillado; e) unguiculado.

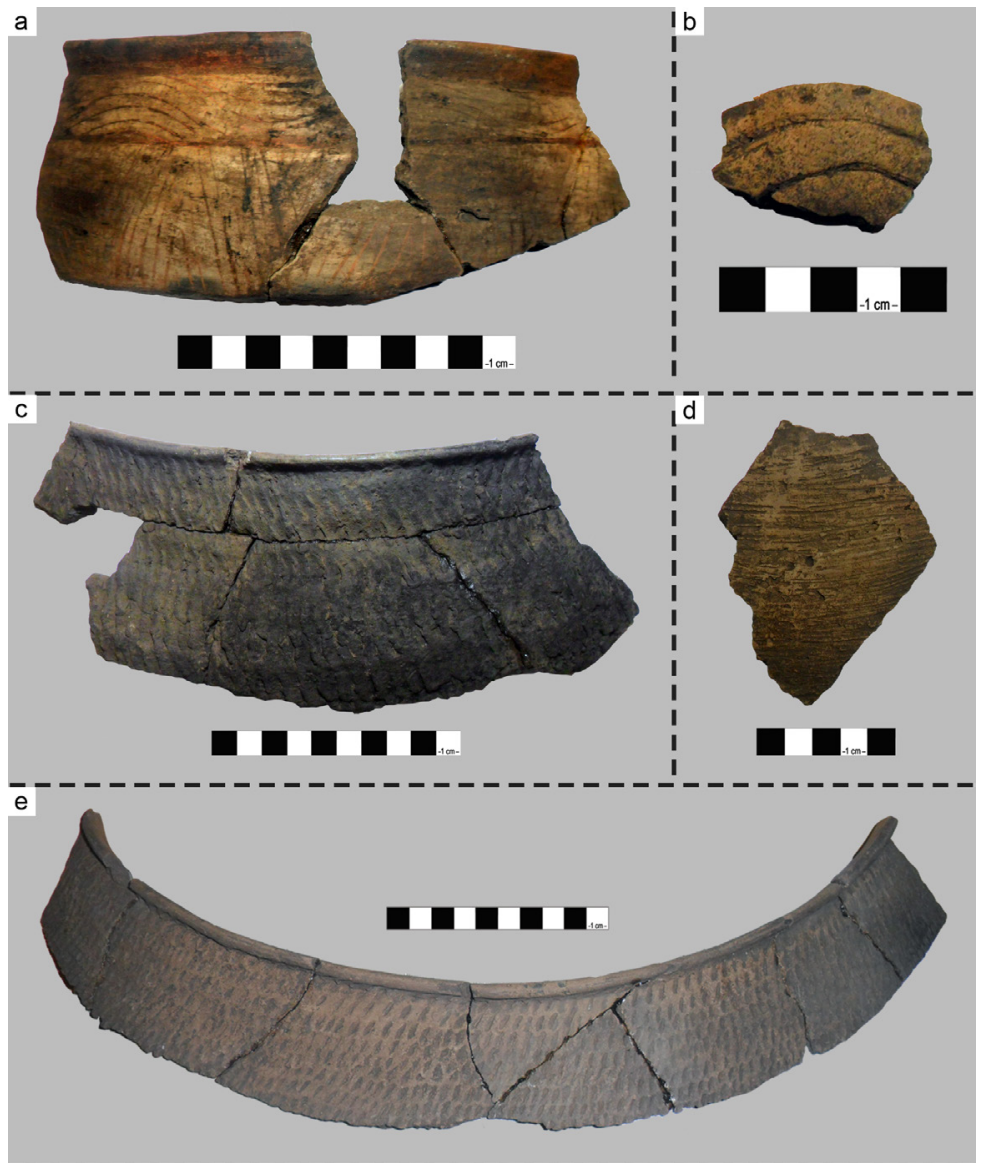

Tabla 2. Porcentajes de las variables tecnológicas/decorativas para FP2 y FP3. En rojo las variables que presentan diferencias significativas entre los conjuntos.

\begin{tabular}{lccccc}
\hline \multirow{2}{*}{$\begin{array}{l}\text { Variables } \\
\text { tecnológicas/decorativas }\end{array}$} & \multicolumn{2}{c}{$\mathrm{FP} 2(\mathrm{~N}=719)$} & \multicolumn{2}{c}{$\mathrm{FP} 3(\mathrm{~N}=1328)$} & \multirow{2}{*}{$\mathrm{X}^{2}$} \\
\cline { 2 - 5 } & $\mathrm{n}$ & $\%$ & $\mathrm{n}$ & $\%$ & \\
\hline Inclusiones & 484 & 67,32 & 931 & 70,11 & $\mathrm{p}=0,1922$ \\
\hline Baño & 349 & 48,54 & 776 & 58,43 & $\mathrm{p}<0,0001$ \\
\hline Pulido & 68 & 9,46 & 297 & 22,36 & $\mathrm{p}<0,0001$ \\
\hline Alisado & 246 & 34,21 & 398 & 29,97 & $\mathrm{p}=0,484$ \\
\hline Unguiculado & 26 & 3,62 & 63 & 4,74 & $\mathrm{p}=0,2323$ \\
\hline Corrugado & 23 & 3,2 & 48 & 3,61 & $\mathrm{p}=0,6238$ \\
\hline Inciso & 39 & 5,42 & 52 & 3,92 & $\mathrm{p}=0,1139$ \\
\hline Pintura & 56 & 7,79 & 209 & 15,74 & $\mathrm{p}=<0,0001$ \\
\hline
\end{tabular}


Por otro lado, en FP2 se registró la presencia de inclusiones en un 67,32\% ( $\mathrm{n}=484)$, mientras que en FP3 en un 70,11\% ( $\mathrm{n}=931)$, observándose principalmente tiesto molido $y$, en menor frecuencia, otros elementos como mica y partículas biosilíceas. Las fracturas relevadas fueron mayormente de carácter irregular $(\mathrm{FP} 2=61,59 \%$ y FP3 $=64,14 \%$ ). Respecto del color de los fragmentos, en ninguno de los sectores se hallaron fragmentos correspondientes al espectro 3. Los predominantes en ambos sectores son el 4 en el núcleo y en las márgenes (porcentajes mayores al 75\%) y el 1 en las superficies (porcentajes mayores al $72 \%$ ), siendo muy escaso el espectro 2 (menor al $1 \%$ en todos los casos) (Tabla 3). En consecuencia, puede inferirse que la atmósfera de cocción es principalmente reductora en ambos sectores (74,9\% en FP3 y $72,5 \%$ en FP2), seguido de oxidante $(17,8 \%$ en FP3 y $16,7 \%$ en FP2) y parcialmente oxidante (7,3\% en FP3 y $10,8 \%$ en FP2).

Tabla 3. Frecuencia de las variables vinculadas al color de los fragmentos para FP2 y FP3

\begin{tabular}{|c|c|c|c|c|c|c|c|c|c|c|c|c|c|}
\hline \multirow{3}{*}{ Color } & \multicolumn{6}{|c|}{ FP2 $(N=719)$} & \multicolumn{6}{|c|}{ FP3 $(\mathrm{N}=1328)$} & \multirow{3}{*}{$\mathrm{X}^{2}$} \\
\hline & \multicolumn{2}{|c|}{1} & \multicolumn{2}{|c|}{2} & \multicolumn{2}{|r|}{3} & \multicolumn{2}{|c|}{1} & \multicolumn{2}{|c|}{2} & \multicolumn{2}{|c|}{4} & \\
\hline & $\mathrm{n}$ & $\%$ & $\mathrm{n}$ & $\%$ & $\mathrm{n}$ & $\%$ & $\mathrm{n}$ & $\%$ & $\mathrm{n}$ & $\%$ & $\mathrm{n}$ & $\%$ & \\
\hline Núcleo & 95 & 13,21 & 4 & 0,56 & 620 & 86,23 & 236 & 17,77 & 0 & 0 & 1092 & 82,23 & $\mathrm{p}=0,1004$ \\
\hline $\begin{array}{l}\text { Margen } \\
\text { Externo }\end{array}$ & 176 & 24,48 & 6 & 0,83 & 537 & 74,69 & 314 & 23,64 & 3 & 0,23 & 1011 & 76,13 & $\mathrm{p}=0,1229$ \\
\hline $\begin{array}{l}\text { Margen } \\
\text { Interno }\end{array}$ & 143 & 19,89 & 3 & 0,42 & 573 & 79,69 & 262 & 19,73 & 0 & 0 & 1066 & 80,27 & $\mathrm{p}=0,0618$ \\
\hline Sup. Externa & 519 & 72,18 & 6 & 0,83 & 194 & 26,98 & 957 & 72,06 & 5 & 0,38 & 366 & 27,56 & $\mathrm{p}=0,3909$ \\
\hline Sup. Interna & 562 & 78,16 & 5 & 0,7 & 152 & 21,14 & 1049 & 78,99 & 2 & 0,15 & 277 & 20,86 & $\mathrm{p}=0,1283$ \\
\hline
\end{tabular}

Los resultados del test de chi cuadrado muestran que de las 22 variables analizadas, 7 presentan diferencias significativas entre los conjuntos (Tablas 1, 2 y 3). De éstas, 4 se vinculan con factores tafonómicos: agrietamiento, manganeso, raíces y roedores y 3 con aspectos tecnológicos/decorativos: baño, pulido y pintura.

\section{Discusión y consideraciones finales}

Uno de los objetivos de este trabajo consistió en ampliar el conocimiento acerca de la alfarería de las poblaciones que habitaron un sector de borde de la Llanura Aluvial del Paraná medio. Considerando la escasez de registros que existen en la región para el momento de contacto europeo-indígena, los datos que provienen del sitio arqueológico FP presentan un aporte significativo para la arqueología en un período temporal poco estudiado.

Para poder interpretar tales resultados se hace necesario, en primera instancia, establecer si los conjuntos procedentes del sitio presentan características similares. En este punto, los resultados obtenidos de los análisis tecnológicos y decorativos 
Balducci, Galligani y Sartori. Caracterización del registro cerámico del sitio arqueológico Familia Primón...

muestran que no presentan diferencias significativas entre sí, excepto en relación con aquellas variables que pueden verse afectadas por una mayor/menor incidencia de los agentes tafonómicos ( $\mathrm{v} . \mathrm{g}$. la presencia de pintura, baño y pulido). En cuanto a estos tres atributos, las frecuencias son mayores en FP3, sector que se halla menos perturbado postdepositacionalmente, cuestión que puede influir en el relevamiento de los diferentes tratamientos superficiales ya que pueden verse afectados por algunos procesos tafonómicos - tales como el agrietamiento, depositación de óxido de manganeso, marcas de raíces- que tienen el potencial de enmascarar o dificultar su identificación. Estos agentes pueden, entonces, explicar, en parte, las diferencias halladas respecto de las variaciones en las frecuencias registradas en cada conjunto.

Por lo tanto, la escasa variabilidad inter-conjunto no puede asignarse al empleo de técnicas distintas para la elaboración y la decoración de la cerámica sino que las diferencias estarían reflejando la microdinámica del ambiente de depositación de ambos conjuntos. Una parte de los tiestos de FP2 se halló expuesta en superficie (o a escasa profundidad) en lo que actualmente es una calle con tránsito vehicular, abierta al público hace unos diez años. Si bien, como se mencionó con anterioridad, los test estadísticos realizados no muestran diferencias significativas entre los fragmentos hallados en superficie y en estratigrafía - probablemente porque el tiempo de exposición fue corto-, se entiende que los primeros se ven más afectados por procesos postdepositacionales como la exposición directa a factores climáticos (v.g. lluvia, humedad, calor) y a diferentes fuerzas de compresión/erosión vinculadas con el tránsito de vehículos y el pisoteo.

Ahora bien, mediante el análisis de los resultados y de su comparación con la información obtenida con otros conjuntos cerámicos - procedentes de sitios arqueológicos de la zona- se pueden observar tanto recurrencias como diferencias en ciertas características del material examinado. A partir del tipo de fractura y del color predominante en los márgenes y en el núcleo de los tiestos - con tonalidades que van del gris al negro- se infiere que la cocción de los contenedores se llevó a cabo a temperatura elevada y en una atmósfera reductora. Este tipo de atmósfera suele estar asociada a cocciones abiertas, donde el combustible y los contenedores se mantienen juntos, y el oxígeno no tiene una libre circulación (Convención Nacional de Antropología, 1966; García Rosselló y Calvo Trias, 2006). También se observa el agregado de tiesto molido, lo que sugiere la intencionalidad de los alfareros de agregar este tipo de material como antiplástico, práctica que en el área se viene desarrollando desde $c a$. $1000 \mathrm{AP}$ (Di Prado, 2018). Estos resultados son compatibles con los alcanzados en FP1 por Feuillet Terzaghi (2009), en otros sitios arqueológicos de la margen santafesina del río Paraná (Coll, 2011; Feuillet Terzaghi et al., 2008; Letieri et al., 2013; Sartori et al., 2013) así como en investigaciones efectuadas sobre la margen izquierda de dicho río (ver Di Prado, 2016; Ottalagano, 2011; Ottalagano, et al., 2015; Píccoli y Barboza, 2013, entre otros).

Por otro lado, en relación a la decoración incisa —que en FP2 y FP3 representa el 5,4\% y el 3,9\% respectivamente-, los porcentajes son equivalentes a otros sitios cercanos (v.g. Las Tejas (LT) y Pajas Blancas (PB)) (Sartori, 2015; Sartori et al., 2013) aunque significativamente menor que en otros emplazados en ambas márgenes del río 
Paraná (ver Balducci et al., 2017; Coll, 2011; Letieri et al., 2013; Ottalagano et al., 2015). El tipo de incisión más implementado en FP -localizado en el sector superior de la vasija, debajo del borde- fue el surco rítmico, el cual es recurrente en los sitios arqueológicos del Nordeste y que comúnmente es adscripto a la entidad arqueológica Goya-Malabrigo (Ceruti, 1986, 2003). Asimismo, debe mencionarse la presencia, en FP3, de un elemento característico de este estilo, como es el apéndice zoomorfo que representa a un psitácido (Figura 2a) (Ceruti, 2003; Loponte y Acosta, 2013) y de restos de alfarería gruesa o campana (sensu Gaspary 1950). Sin embargo, la presencia del modelado es escasa tanto en FP como en los demás conjuntos de la zona de la laguna Coronda, destacándose en LT un apéndice asignable a carpincho (Hydrochoerus hydrochaeris) y uno a yaguareté (Panthera onca) en Los Bañados (LB).

Otros aspectos decorativos que se diferencian de los ya mencionados son la presencia de corrugado, unguiculado y pintura bicrómica - finas líneas rojas sobre fondo blanco-, los cuales sumados representan un 7,23\% y un $8,96 \%$ del total en FP2 y en FP3, respectivamente (Figuras 3a, 3c y 3e). Éstos han sido tradicionalmente asignados a la unidad arqueológica o entidad cultural Guaraní (Bonomo, 2012; Brochado, 1973; Loponte y Acosta, 2013; Serrano, 1931, entre muchos otros), al igual que las técnicas de cepillado y roletado (sensu Chmyz, 1976), que se hallan en escasa frecuencia en ambos conjuntos (Figura 3b y 3d). Respecto del empleo de corrugado y unguiculado, es inusual en los sitios del Paraná medio, aunque se registran en mayores porcentajes en la zona del delta de este río (promedios de $32,8 \%$ y $7 \%$, respectivamente; Loponte y Acosta, 2013). Si bien algunos autores sostienen que el corrugado pudo haber sido utilizado por grupos cazadores-recolectores locales (Ceruti y González, 2007; Frère, 2015), se considera necesario evaluar los hallazgos en el contexto general del sitio para una correcta interpretación. Por su parte, Loponte y Acosta (2013) señalan que el unguiculado, en el caso de los grupos locales, se halla generalmente combinado con típicos motivos geométricos incisos. En este punto, cabe aclarar que en FP2 y FP3 ninguno de los tiestos unguiculados se encuentra asociado con decoración incisa. Además, es remarcable la presencia de diferentes variantes de corrugado identificadas en ambos sectores, la mayor parte de las cuales se exhibe en su cara externa, junto con alisado en su superficie interna.

En general, los atributos asignables a la unidad arqueológica Guaraní se asemejan a los mencionados para el sitio Cerro de las Pajas Blancas 1, el cual también se localiza en el Paraná medio, a poca distancia de FP y cuenta con una cronología similar, proveniente de tres dataciones radiocarbónicas: $506 \pm 43$ (Sartori, 2013), 640 \pm 60 y $650 \pm 70$ años AP (Bonomo et al., 2011). En este sitio, Badano (1940) recuperó una urna funeraria (pieza $n^{\circ} 13$ ) pintada de rojo sobre fondo blanco, asignable a la unidad Guaraní. En el caso de FP, la frecuencia de materiales asociados a esta unidad arqueológica no es suficiente para sostener una ocupación efectiva de los grupos humanos comúnmente vinculados con aquella. En este sentido, en el área de entierros (FP1) no se hallaron inhumaciones en urnas, característica típica de esta unidad (Ambrosetti, 1985), además de que se observa un predominio de la cerámica local en el conjunto y de que los 
Balducci, Galligani y Sartori. Caracterización del registro cerámico del sitio arqueológico Familia Primón...

recursos arqueofaunísticos y materiales líticos indican patrones también asimilables a los asignados a grupos locales.

No obstante, la presencia de ese estilo cerámico en el sitio sí sugiere un probable contacto con grupos guaraníes, cuya expansión desde el norte de Brasil hacia el río de la Plata es un tópico ampliamente abordado en la literatura arqueológica (v.g. Bonomo, 2012; Brochado, 1984; Loponte y Acosta, 2013; Noelli, 2004). Hasta el momento, el modelo más aceptado sugiere el ingreso de estas poblaciones hacia el actual territorio argentino por el río Uruguay, pocos siglos antes de la llegada de los conquistadores europeos y más tardíamente hacia la zona del Paraná inferior y el delta (Bonomo, 2012; Loponte y Acosta, 2008). Este planteo se refuerza con el hecho de que la mayoría de los sitios vinculados con características guaraníes se localizan en las inmediaciones de la desembocadura del río Uruguay, en el estuario del río de la Plata, mientras que su presencia disminuye hacia el Paraná medio (Bonomo, 2012; Loponte y Acosta, 2013). En general, se acepta que estas poblaciones no ocuparon el tramo medio del río Paraná por la competencia - por el espacio - con los grupos cazadores-recolectorespescadores locales (Rodríguez, 2004) y el hallazgo de fragmentos cerámicos con rasgos de esta unidad arqueológica en ciertos sitios de este tramo (v.g. FP y PB) se atribuye al comercio, circulación e intercambio (Bonomo, 2012; Feuillet Terzaghi, 2009).

Por último, se destaca que los fechados radiocarbónicos disponibles para FP y PB se encuentran dentro del rango temporal de los sitios con contextos guaraníes situados en el delta del río Paraná (v.g. Arroyo Fredes, Arroyo Malo, Arena Central, El Arbolito) (ver Bonomo et al., 2015; Loponte y Acosta, 2013) y que se sitúan en momentos cercanos al contacto europeo-indígena. En este punto, cabe mencionar que diferentes fuentes históricas señalan la presencia de grupos guaraníes en las islas cercanas al fuerte Sancti Spiritus (v.g. Ramírez, 2007 [1528]; García de Moguer, 1908 [1526]), en la confluencia de los ríos Carcarañá y Coronda, a escasa distancia de FP. De igual modo, las evidencias halladas en el sitio Sancti Spiritus muestran, además de la presencia europea, ciertos rasgos guaraníes como el corrugado y el unguiculado (en porcentajes cercanos al 1\%), asociados a otros asignables al tipo Goya-Malabrigo (Letieri et al., 2013). Por lo tanto, es posible que, dadas las condiciones, se haya producido algún tipo de interacción entre las diferentes parcialidades locales con los grupos guaraníes.

En síntesis, de este primer análisis se observan, por un lado, algunas tendencias en el material cerámico que se correlacionan con los resultados obtenidos en otros sitios cercanos a FP, comúnmente vinculados a contextos Goya-Malabrigo (v.g. presencia de apéndices zoomorfos, surco rítmico, alfarería gruesa) y, por otro lado, determinadas características relacionadas a la unidad arqueológica Guaraní (v.g. corrugado, unguiculado), poco frecuentes en el área de estudio. En este sentido, es necesario continuar evaluando el registro cerámico, mediante estudios más específicos ( $v . g$. cortes petrográficos), para profundizar en sus particularidades y en los modos de hacer de los grupos humanos que habitaron el área en el pasado. A su vez, es preciso vincularlo con otro tipo de evidencias arqueológicas halladas en FP - y en otros sitios del área-, para ampliar el conocimiento acerca de la ocupación del espacio y del tipo de relaciones 
establecidas entre las poblaciones que lo habitaban, en un momento de complejidad en el cual no sólo se produjo la llegada de los europeos, sino también la de grupos originarios de otras regiones geográficas.

\section{Agradecimientos}

Queremos agradecer a la Fundación Arqueológica del Litoral (FUNDARQ), a la Municipalidad de Coronda y al Museo Municipal "José Manuel Maciel" por su constante apoyo a la investigación. A Claudio Ñañez y a toda la comunidad Corondá por su buena predisposición y por su aval para realizar trabajos arqueológicos. A los habitantes del barrio Gálvez por recibirnos en cada campaña. A todos los integrantes del Grupo de Investigaciones Arqueológicas del Nordeste (GIAN) por su colaboración en los análisis para este trabajo.

\section{Referencias bibliográficas}

Ambrosetti, J. B. (1895). "Los cementerios prehistóricos del Alto Paraná (Misiones)". Boletín del Instituto Geográfico Argentino, 16, 227-263.

Amitrano Bruno, R. (1984). "Informe sobre el tratamiento de restauración de una pieza cerámica del yacimiento ibérico de 'El Amarejo"'. Revista Al-Basit, 15, 173-185.

Badano, V. M. (1940). Piezas Enteras de Alfarería del Litoral Existentes en el Museo de Entre Ríos. Paraná: Editorial Predassi.

Balducci, F. (2014). "Ocupaciones humanas del Holoceno tardío: estudio arqueopalinológico del sitio Familia Primón (provincia de Santa Fe)". Tesis de Licenciatura en Antropología, Facultad de Humanidades y Artes, Universidad Nacional de Rosario, Rosario.

Balducci, F.; Riberi, M. y F. Lancellotti. (2017). Análisis de la decoración incisa en la alfarería del sitio arqueológico (departamento San Jerónimo, Santa Fe, Argentina). Scientia Interfluvius, suplemento resúmenes VII EDAN, 68.

Balfet, H.; Fauvet Berthelot, M. y S. Monzón. (1992). Normas para la descripción de vasijas cerámicas. México: Centre D’Études Mexicaines et Centraméricaines.

Banning, E. (2000). The Archaeologist's laboratory. The Analysis of Archaeological Data. New York: Plenum Publishers.

Barrientos, G. (1997). "Nutrición y dieta de las poblaciones aborígenes prehispánicas del sudeste de la Región Pampeana". Tesis Doctoral en Ciencias Naturales, Facultad de Ciencias Naturales y Museo, Universidad Nacional de La Plata, La Plata.

Beck, M.; Skibo, J.; Hally, D. y P. Yang. (2002). "Sample selection for ceramic usealteration analysis: The effects of abrasion on soot". Journal of Archaeological Science, 29(1), 1-15.

Behrensmeyer, A. (1978). "Taphonomic and ecological information from bone weathering". Paleobiology, 4(2), 150-162. 
Balducci, Galligani y Sartori. Caracterización del registro cerámico del sitio arqueológico Familia Primón...

Bennàsar Serra, M. (2010). “Tafonomía de micromamíferos del Pleistoceno Inferior de la Sierra de Atapuerca (Burgos): la Sima del Elefante y la Gran Dolina". Tesis Doctoral, Universitat Rovira i Virgili, Tarragona.

Bonomo, M. (2012). Historia Prehispánica de Entre Ríos. Buenos Aires: Fundación de Historia Natural Félix de Azara.

Bonomo, M.; Costa Angrizani, R.; Apolinaire, E. y F. Silva Noelli. (2015). "A model for the Guaraní expansion in the La Plata Basin and littoral zone of southern Brazil". Quaternary International 356: 54-73.

Bonomo, M.; Politis, G.; Silva, C.; Bastourre, L.; Ramos van Raap, M. A.; Castiñeira Latorre, C.; Scabuzzo, C. y E. Apolinaire. (2017). "Estado actual de las investigaciones en la localidad arqueológica Laguna de los Gansos (Diamante, Entre Ríos)". Revista del Museo de Antropología, 9 (2), 51-66.

Bonomo, M.; Politis, G. y C. Gianotti García. (2011). "Montículos, jerarquía social y horticultura en las sociedades indígenas del Delta del río Paraná (Argentina)". Latin American Antiquity, 12(2), 167-81.

Brochado, J. (1973). "Migraciones que difundieron la tradición alfarera Tupiguaraní". Relaciones de la Sociedad Argentina de Antropología, 7, 7-39.

Brochado, J. (1984). "An Ecological Model of the Spread of Pottery and Agriculture into Eastern South America". Tesis Doctoral, University of Illinois, UrbanaChampaing.

Burkart, R.; Bárbaro, N.; Sánchez, R. y D. Gómez (1999). Ecorregiones de la Argentina. Buenos Aires: Administración de Parques Nacionales.

Ceruti, C. (1986). "Algo sobre crítica y autocrítica en arqueología". Revista de Antropología, 1(1), 19-24.

Ceruti, C. (2003). "Entidades culturales presentes en la cuenca del Paraná Medio (margen entrerriana)". Mundo de Antes, 3, 111-135.

Ceruti, C. y González, M. I. (2007). "Modos de vida vinculados con ambientes acuáticos del Nordeste y Pampa bonaerense de Argentina". Relaciones de la Sociedad Argentina de Antropología, 32, 101-140.

Chmyz, I. (1976). "Terminologia arqueológica brasileira para a cerámica". Cadernos de Arqueologia, 1(2), 119-148.

Cocco, G. (1996). "Procesos de formación y transformación de sitios arqueológicos en el sector sur del bajo de los Saladillos: sitio Isla Barranquita". Tesis de Licenciatura en Antropología, Facultad de Humanidades y Artes, Universidad Nacional de Rosario, Rosario.

Coll, M. (2011). "Análisis de la tecnofactura del material cerámico arqueológico del sitio "Playa Mansa" (provincia de Santa Fe). Resultados preliminares". En Ma. R. Feuillet Terzaghi, Ma. B. Colasurdo, J. Sartori y S. Escudero (eds.), Avances y perspectivas en arqueología del nordeste (69-85). Buenos Aires: Editorial El Talar.

Convención Nacional de Antropología. (1966). Primera Convención Nacional de Antropología. Córdoba: Universidad Nacional de Córdoba.

Cremonte, M. B. y Bugliani, M. F. (2006-2009). "Pastas, formas e iconografía. Estrategias para el estudio de la cerámica arqueológica". Xama, 19/23, 239-262. 
Di Prado, V. (2016). "Primeras etapas de elaboración cerámica en Los Tres Cerros 1 (Victoria, Entre Ríos). Caracterización mediante petrografía de pastas". Intersecciones en Antropología - Volumen especial, 3, 23-32.

Di Prado, V. (2018). "Prácticas alfareras prehispánicas y procesos de Interacción social en el centro-este de argentina Durante el holoceno tardío". Latin American Antiquity, 29(3), 552-571.

Di Rienzo J.A.; Casanoves, F., Balzarini, M.G., González, L., Tablada M. y C. W. Robledo. (2016). InfoStat versión 2016. Grupo InfoStat, FCA, Universidad Nacional de Córdoba, Argentina. URL http://www.infostat.com.ar

Escudero, C. S. (1996). "Evaluación de contexto de depositación en ambientes fluviales: Propuesta de un modelo y su aplicación al sitio Bajada Guereño (provincia de Santa Fe)". Tesis de Licenciatura en Antropología, Facultad de Humanidades y Artes, Universidad Nacional de Rosario, Rosario.

Fantuzzi, L. (2010). "La alteración posdeposicional del material cerámico. Agentes, procesos y consecuencias para su preservación e interpretación arqueológica". Comechingonia virtual, 4(1), 27-59.

Feuillet Terzaghi, M. R. (2009). "El registro bioarqueológico de la cuenca inferior del río Salado y cuenca del río Coronda (Margen derecha, provincia de Santa Fe)". Tesis de Doctoral en Humanidades y Artes, Facultad de Humanidades y Artes, Universidad Nacional de Rosario, Rosario.

Feuillet Terzaghi, M. R.; Deluca, S. y D. Ivarz. (2008). Nuevos aportes al estudio de la cerámica del Sitio Isleta del Árbol Viejo (dpto. San Jerónimo, pcia. de Santa Fe-Argentina). En: Actas del XXVIII Encuentro de Geohistoria Regional (pp. 349-354). Resistencia: Instituto de Investigaciones Geohistóricas, CONICET.

Frère, M. M. (2015). "Tecnología cerámica de los cazadores-recolectores-pescadores de la microrregión del río Salado, provincia de Buenos Aires". Tesis Doctoral en Antropología, Facultad de Filosofía y Letras, Universidad de Buenos Aires, Buenos Aires.

Galligani, P. y Balducci, F. (2014). Aspectos decorativos de la producción cerámica durante el holoceno tardío en el sitio Familia Primón (Coronda, Santa Fe). En: Libro de Resúmenes VII Congreso de Arqueología de la Región Pampeana Argentina, CDROM. Rosario.

Galligani, P.; Balducci, F.; Sartori, J. y M. Riberi. (2018). “Análisis del registro cerámico desde una perspectiva tafonómica: el caso de los sitios arqueológicos Familia Primón y Los Bañados (centro-este de Santa Fe, Argentina)". Revista Mundo de Antes, 12(1), 107-133.

García de Moguer, D. (1908) [1526]. "Relación y derrotero de Diego García". En J. Medina (comp.) Los viajes de Diego García de Moguer al Río de la Plata (232246). Santiago de Chile: Imprenta Elzeviriana.

García Rosselló, J. y Calvo Trias, M. (2006). “Análisis de las evidencias macroscópicas de cocción en la cerámica prehistórica: una propuesta para su estudio". Mayurqa, $31,83-112$ 
Gaspary, F. (1950). "Investigaciones arqueológicas y antropológicas en un "cerrito" de la isla Los Marinos (Dto. Victoria, Entre Ríos) situada frente a Rosario". Publicaciones del Instituto de Arqueología, Lingüística y Folclore, 23, 3-66.

González, M. I. y Frère, M. M. (2010). Diseños prehispánicos de la Alfarería pampeana. Buenos Aires: Editorial de la Facultad de Filosofía y Letras.

Gutiérrez, M. (2004). "Análisis tafonómicos en el área interserrana (provincia de Buenos Aires)". Tesis Doctoral en Ciencias Naturales, Facultad de Ciencias Naturales y Museo, Universidad Nacional de La Plata, La Plata.

Hally, D. (1983). "Use alteration of pottery vessel surfaces: an important source of evidence for the identification of vessel function". North American Archaeologist, 4, 3-26.

Hogg, A.; Hua, Q.; Blackwell, P.G.; Buck, C. E.; Guilderson. T. P.; Heaton, T.J.; Niu, M.; Palmer, J. G.; Reimer, P. J.; Reimer, R. W.; Turney, C. y S. Zimmerman. (2013). "SHCAL13 Southern Hemisphere calibration, 0-50,000 years Cal. BP". Radiocarbon, 55(4), 1889-1903.

Iribarne, E. (1937). "Algunos vasos indígenas de las márgenes del Paraná inferior". Relaciones de la Sociedad Argentina de Antropología, 1, 181-190.

Lafón, C. (1972). "El replanteo para la arqueología del nordeste argentino". Antiquitas, 16, 1-16.

Lemonnier, P. (1992). Elements for an Anthropology of Technology. Ann Arbor, Michigan.

Letieri, F. (1999). "Análisis tecnocomparativo del material cerámico arqueológico en el extremo oriental del Área con Paleocauces Enterrados de la provincia de Santa Fe". En: Actas del XIX Encuentro de Geohistoria Regional (297-308). Corrientes.

Letieri, F.; Cocco, G.; de la Fuente, G. A.; Meletta, H. y C. Alberico. (2013). "La variabilidad y complejidad artefactual de la producción alfarera procedente del área de estudio correspondiente al primer asentamiento europeo en la cuenca del Río de La Plata: Fuerte Sancti Spiritus (1527-1529) - Un abordaje interdisciplinario". En: Actas del V Congreso Nacional de Arqueología Histórica (Tomo 2) (464-490). Saarbrucken, Alemania.

Letieri, F.; Cocco, G.; Frittegotto, G. y I. Sánchez Pinto. (2004). "El fuerte Sancti Spiritus, el primer asentamiento europeo en el actual territorio argentino". Revista Ciencia Hoy, 24(142), 13-18.

Loponte, D. y Acosta, A. (2008). "Estado actual y perspectivas de la arqueología de la Tradición Tupiguaraní en Argentina”. En T. Andrade Lima y A. Prous (eds.), Os Ceramistas Tupiguarani, vol. I (197-215). Belo Horizonte: Sigma.

Loponte, D. y Acosta, A. (2013). "La construcción de la unidad arqueológica guaraní en el extremo meridional de su distribución geográfica". Cuadernos del Instituto Nacional de Antropología y Pensamiento Latinoamericano - Series Especiales, $1(4), 193-235$.

Noelli, F. S. (2004): "La distribución geográfica de las evidencias arqueológicas Guaraní (Brasil, Argentina, Uruguay y Paraguay)". Tellus, 4(7), 15-36.

Ottalagano, F. (2010) "Decoración experimental de cerámica aplicada al estudio de las técnicas incisas del área del Paraná”. Intersecciones en Antropología, 11(2), 237-247. 
Ottalagano, F. (2011). "Análisis contextual del registro artístico del sitio A. Arenal 1 (Provincia de Entre Ríos, Argentina): aportes para el estudio de los aspectos identitarios de los grupos humanos del litoral fluvial del Paraná". En C. Páez y G. De la Fuente (eds.). La cerámica en la Materialización de la Sociedad: Transformaciones, Metáforas y Reproducción Social, (125-140). Oxford: BARInternational Series 2294.

Ottalagano, F. (2015). "Explorando la Variabilidad Petrográfica entre Vasijas Decoradas y Lisas del Sitio La Palmera 2 (Entre Ríos, Argentina): Hacia un Abordaje Microscópico del Arte Cerámico Prehispánico de la Cuenca del Río Paraná". Revista Chilena de Antropología, 32(2), 57-68.

Ottalagano, F. (2016). "A diachronic study of pre-Hispanic vessels from the middle basin of Paraná River (South America) using a petrographic approach". Journal of Archaeological Science: Reports, 9, 320-329.

Píccoli, C. y Barboza, C. (2013). "Caracterización del conjunto cerámico recuperado en las actividades de prospección realizadas en el sitio Los Bananos (Goya, Corrientes, Argentina)". Intersecciones en Antropología, 14, 213-225.

Ramírez, L. (2007) [1528]. "Carta de Luis Ramírez a su padre desde el Brasil (1528): orígenes de lo "real maravilloso" en el Cono Sur. Introducción y notas de J. F. Maura". Recuperado de <http://parnaseo.uv.es/Lemir/Textos/Ramirez.pdf>.

Revised Standard soil Color Charts (2001). Fujihira Industry Company.

Rice, P. M. (1987). Pottery Analysis: A Sourcebook. Chicago: The University of Chicago Press. Rodríguez, J. (2004). "En busca de la tierra sin mal. El poblamiento de la cuenca del Plata por los guaraníes prehistóricos". Ciencia Hoy, 14(80), 28-33.

Rye, O. (1981). Pottery Technology: Principles and Reconstruction. Washington DC: Taraxacum.

Sanhueza Riquelme, L. (1998). "Antecedentes y proposición metodológica para el estudio de huellas de alteración en la cerámica". Conserva, 2, 69-79.

Sartori, J. (2008). "La cuenca inferior del río Salado: un desafío para los análisis arqueofaunísticos". Tesis de Licenciatura en Antropología, Facultad de Humanidades y Artes, Universidad Nacional de Rosario, Rosario.

Sartori, J. (2013). "Variabilidad arqueofaunística en la cuenca media e inferior del río Coronda". Tesis Doctoral en Humanidades y Artes, Facultad de Humanidades y Artes, Universidad Nacional de Rosario, Rosario.

Sartori, J. (2015). "Pasado y presente de las investigaciones Arqueológicas en el sitio Las Tejas (Santa Fe, Argentina)". Revista de Antropología del Museo de Entre Ríos, 1(2), 109-120.

Sartori, J.; Balducci, F. y P. Galligani. (2018). "Primeras evidencias de macrorrestos vegetales en el sitio arqueológico Familia Primón (Santa Fe, Argentina)". Revista de Arqueología Iberoamericana, 37, 48-52.

Sartori, J.; Scuizzatto, F. y P. Galligani. (2013). "Análisis comparativo de la tecnomorfología cerámica de los sitios de la cuenca media e inferior del río Coronda". Cuadernos del Instituto Nacional de Antropología y Pensamiento Latinoamericano, Series Especiales, 1(2), 107-119. 
Schiffer, M. y Skibo, J. (1989). "A provisional theory of ceramic abration". American Anthropologist, 91(1), 101-115.

Serrano, A. (1923). "Arqueología de Las Tejas (provincia de Santa Fe)". Revista Universitaria del Litoral, 12,15-64.

Serrano, A. (1931). "Arqueología del Litoral”. Memorias del Museo de Paraná, 4, 4-15.

Serrano, A. (1972). Lineas fundamentales de la arqueología del Litoral (una tentativa de periodización). Córdoba: Instituto de Antropología.

Skibo, J. (1987). "Fluvial sherd abrasion and the interpretation of surface remains on Southwestern bajadas". North American Archaeologist, 8, 125-142.

Skibo, J. (1992). Pottery Function: a Use-Alteration Perspective. New York: Plenum Press.

Skibo, J. y Schiffer, M. (1987). "The effects of water on processes of ceramic abrasion". Journal of Archaeological Science, 14, 83-96.

Stuiver, M.; Reimer, P. y R. Reimer. (2005). Calib Radiocarbon Calibration version 7.0.2: http://calib.qub.ac.uk/calib/index.html. (Acceso 14 de agosto, 2014).

Tschegg, C. (2009). "Post-depositional surface whitening of ceramic artifact: alteration mechanisms and consequences". Journal of Archaeological Science, 36, 2155-2161.

Ward, G. y Wilson, S. (1978). "Procedures for Comparing and Combining Radiocarbon Age-Determinations - Critique". Archaeometry, 20, 19-31. 